\title{
The Hafele-Keating Circumnavigating Atomic Clock Experiment and the Newton-Voigt Relativistic Space-time Transformation
}

Robert J. Buenker ${ }^{1 *}$

${ }^{1}$ Fachbereich C-Mathematik und Naturwissenschaften, Bergische Universität Wuppertal, Gaussstr. 20, D-42097 Wuppertal, Germany

\begin{tabular}{|c|c|}
\hline $\begin{array}{c}\text { Article History } \\
\text { Received: } 13.03 .2021 \\
\text { Accepted: } 19.04 .2021 \\
\text { Published: } 26.04 .2021 \\
\\
\text { Journal homepage: } \\
\text { https://www.easpublisher.com }\end{array}$ & $\begin{array}{l}\text { Abstract: The Lorentz transformation (LT) has been shown to be self-contradictory (Clock } \\
\text { Puzzle). The reason is because it predicts both proportional time dilation and the remote } \\
\text { non-simultaneity of events. Consequently, the LT erroneously requires that when a null } \\
\text { time difference is multiplied with a constant time-dilation factor, the resulting time } \\
\text { difference in another rest frame can be different from zero. This possibility stands in direct } \\
\text { conflict with a fundamental axiom of elementary algebra. The precursor of the LT was } \\
\text { introduced by Voigt in } 1887 \text {. He pointed out that one can make a change in the Galilean } \\
\text { transformation that makes it compatible with experimental data which indicate that the } \\
\text { speed of light in free space is the same for all observers, regardless of their state of motion. } \\
\text { The history of how the Voigt transformation ultimately evolved into the LT over the next } 20 \\
\text { years is reviewed. In the process, it is shown how a different approach can be taken which } \\
\text { eliminates the above contradiction inherent in the LT, while at the same time retaining the } \\
\text { essential characteristics of light-speed constancy and consistency with Galileo's Relativity } \\
\text { Principle (RP). The resulting space-time transformation eschews the space-time mixing } \\
\text { characteristic of both the LT and the original Voigt transformation. In this way, it removes } \\
\text { the possibility of remote non-simultaneity, and instead insists on the proportionality of time } \\
\text { dilation, which in turn is shown to follow directly from a straightforward extension of } \\
\text { Newton's Law of Inertia. The resulting set of equations [Newton-Voigt Transformation } \\
\text { (NVT)] is found to agree with all experimental timing data obtained to the present day. } \\
\text { Keywords: Voigt Transformation, Time Dilation, Remote Non-simultaneity, Clock Puzzle, } \\
\text { Lorentz Transformation (LT), Universal Time-dilation Law (UTDL), Newton-Voigt } \\
\text { Transformation (NVT). }\end{array}$ \\
\hline
\end{tabular}

Copyright $\odot 2021$ The Author(s): This is an open-access article distributed under the terms of the Creative Commons Attribution 4.0 International License (CC BY-NC 4.0) which permits unrestricted use, distribution, and reproduction in any medium for non-commercial use provided the original author and source are credited.

\section{INTRODUCTION}

The Michelson-Morley experiment [1] employing their newly developed interferometer revived an already longstanding debate among physicists regarding observations of the speed of light in free space [2]. In the same year, Voigt published an article [3] that attempted to explain the apparent independence of the light speed on the state of motion of the observer by making a straightforward change to the classical (Galilean) space-time transformation.

\section{PERFECTING THE VOIGT TRANSFORMATION}

The transformation introduced by Voigt [1] relates the space and time measurements of one observer $(\mathrm{x}, \mathrm{y}, \mathrm{z}, \mathrm{t})$ of a single object with the corresponding values $\left(x^{\prime}, y^{\prime}, z^{\prime}, t^{\prime}\right)$ obtained by a counterpart who is moving with constant speed $\mathrm{v}$ along their mutual $\mathrm{x}, \mathrm{x}^{\prime}$ axis. The result is given below in terms of four equations ( $c$ is the speed of light in free space, $299792458 \mathrm{~ms}^{-1}$ ):

$$
\begin{aligned}
& \mathrm{t}^{\prime}=\mathrm{t}-\mathrm{vc}^{-2} \mathrm{x} \\
& \mathrm{x}^{\prime}=\mathrm{x}-\mathrm{vt} \\
& \mathrm{y}^{\prime}=\gamma^{-1} \mathrm{y} \\
& \mathrm{z}^{\prime}=\gamma^{-1} \mathrm{z}
\end{aligned}
$$

Where $\gamma=\left(1-\mathrm{v}^{2} \mathrm{c}^{-2}\right)^{0.5}$ [exponent should be -0.5]. It is easy to show that both observers agree that the speed of a light pulse has the same value of $\mathrm{c}$ for both of them. This can be done by forming the following linear combination of the two sets of variables from the above equations, namely: 


$$
\left(\mathrm{x}^{2}+\mathrm{y}^{2}+\mathrm{z}^{2}-\mathrm{c}^{2} \mathrm{t}^{2}\right)=\gamma^{-2}\left(\mathrm{x}^{\prime 2}+\mathrm{y}^{\prime 2}+\mathrm{z}^{\prime 2}-\mathrm{c}^{2} \mathrm{t}^{2}\right) \text {. }
$$

In other words, if the speed of the object is measured to be $\mathrm{c}$ in the unprimed rest frame, therefore resulting in a null value on the left-hand side of eq. (2), it also must have a value of $\mathrm{c}$ on the right-hand side.

There are two key aspects of the above transformation which deserve special attention. First, the $\mathrm{x}$-dependent term in eq. (1a) differs from the corresponding $\mathrm{t}=\mathrm{t}^{\prime}$ equation in the Galilean transformation. This change was critical in Voigt's analysis since it leads directly to the desired light-speed constancy requirement otherwise missing from the classical transformation. This is the origin of the wellknown concept of theoretical physics known as spacetime mixing.

Secondly, the $\gamma^{-1}$ factor in both eqs. (1c-1d) also distinguishes the Voigt space-time transformation from the original Galilean transformation. It is this factor which caused his contemporaries to reject his suggestion. The problem lies in the obvious fact that neither of these equations is consistent with Galileo's Relativity Principle, which states that the laws of physics must be independent of the state of motion of the observer. Specifically, the inverse of eq. (1c) is clearly: $y=\gamma y^{\prime}$. That means that the two observers must use a different factor $\left(\gamma\right.$ or $\left.\gamma^{-1}\right)$ to convert the result obtained by one of them to that of the other. Since the two rest frames are considered to be indistinguishable, this is an unacceptable feature of the transformation.
There is another way to see the same point, namely to apply what is generally referred to as Galilean inversion to eqs. (1c-1d). Accordingly, it is argued on the basis of the RP that the inverse of a given equation can be obtained by simulating the exchange of positions of the two observers. This is done by changing the sign of $\mathrm{v}$ and interchanging the primed and unprimed variables therein. When Galilean inversion is applied to eq. (1c), for example, the result is: $y=\gamma^{-1} y^{\prime}$ since $\gamma$ is an even function of v. Combining this equation with eq. (1c) leads to the nonsensical result of $y^{\prime}=\gamma^{-2} y^{\prime}$, again showing that there is something fundamentally wrong with the Voigt transformation.

In retrospect, it is clear where Voigt's otherwise logical argumentation went astray. He overlooked the fact that it is not possible to uniquely specify the desired space-time transformation on the basis of the light-speed constancy assumption by itself. The reason is that speed in general is defined as a ratio of space and time intervals. As a result, one can multiply both the distance $\Delta \mathrm{r}$ traveled by an object and the corresponding elapsed time $\Delta \mathrm{t}$ by the same constant without affecting the value of the its speed.

Lorentz made this point a decade after Voigt's work had appeared $[4,5]$. On this basis one can define a more general transformation than Voigt proposed which also satisfies the light-speed constancy requirement, but without coming into conflict with the $\mathrm{RP}$ (intervals $\Delta \mathrm{x}=\mathrm{x}_{2}-\mathrm{x}_{1}, \Delta \mathrm{x}^{\prime}=\mathrm{x}_{2}^{\prime}-\mathrm{x}_{1}^{\prime}$, etc. are used to emphasize that speeds can only be computed on the basis of such differences):

$$
\begin{aligned}
& \Delta \mathrm{t}^{\prime}=\chi\left(\Delta \mathrm{t}-\mathrm{vc}^{-2} \Delta \mathrm{x}\right)=\chi \eta^{-1} \Delta \mathrm{t} \\
& \Delta \mathrm{x}^{\prime}=\chi(\Delta \mathrm{x}-\mathrm{v} \Delta \mathrm{t}) \\
& \Delta \mathrm{y}^{\prime}=\chi \gamma^{-1} \Delta \mathrm{y} \\
& \Delta \mathrm{z}^{\prime}=\chi \gamma^{-1} \Delta \mathrm{z}
\end{aligned}
$$

with $\eta=\left(1-\mathrm{vc}^{-2} \frac{\Delta \mathrm{x}}{\Delta \mathrm{t}}\right)^{-1} \cdot \quad$ This set of equations differs from the original in eqs. (1a-d) only in that each of the former's right-hand sides is multiplied with the constant factor $\chi$. Forming the analogous linear combination of space and time variables as in eq. (2) then gives:

$$
\begin{aligned}
&\left(\Delta \mathrm{x}^{2}+\Delta \mathrm{y}^{2}+\Delta \mathrm{z}^{2}-\mathrm{c}^{2} \Delta \mathrm{t}^{2}\right)=\gamma^{-2} \chi^{2}\left(\mathrm{x}^{\prime 2}+\mathrm{y}^{\prime 2}+\mathrm{z}^{\prime 2}-\mathrm{c}^{2} \mathrm{t}^{\prime 2}\right) \\
& \\
& \Delta \mathrm{t}^{\prime}=\gamma\left(\Delta \mathrm{t}-\mathrm{vc} \mathrm{c}^{-2} \Delta \mathrm{x}\right)=\gamma \eta^{-1} \Delta \mathrm{t} \\
& \Delta \mathrm{x}^{\prime}=\gamma(\Delta \mathrm{x}-\mathrm{v} \Delta \mathrm{t}) \\
& \Delta \mathrm{y}^{\prime}=\Delta \mathrm{y} \\
& \Delta \mathrm{z}^{\prime}=\Delta \mathrm{z}
\end{aligned}
$$

which confirms that the light-speed constancy requirement is also satisfied by eqs. (3a-3d).

Lorentz [6] and Larmor [7] used this degree of freedom to overcome Voigt's problem with the RP while still adhering to the light-speed constancy. The resulting set of equations is generally referred to as the Lorentz Transformation (LT):
Comparison with the generalized Voigt transformation (GVT) in eqs. (3a-3d) shows that the LT can be obtained from it by simply setting $\chi=\gamma$ therein. The corresponding linear combination of squared intervals for the LT is thus:

$$
\left(\Delta \mathrm{x}^{2}+\Delta \mathrm{y}^{2}+\Delta \mathrm{z}^{2}-\mathrm{c}^{2} \Delta \mathrm{t}^{2}\right)=\left(\Delta \mathrm{x}^{\prime 2}+\Delta \mathrm{y}^{\prime 2}+\Delta \mathrm{z}^{\prime 2}-\mathrm{c}^{2} \Delta \mathrm{t}^{\prime 2}\right),
$$

Thereby also verifying that this set of equations satisfies the light-speed constancy condition. 
Moreover, the inverse set of equations for the LT is obtained directly by applying Galilean inversion to each of eqs. (5a-d), thereby proving that the LT is also perfectly consistent with the RP.

\section{Iii. The clock puzzle and the law of inertia}

There is nonetheless a disqualifying property of the LT. It is obvious from eq. (5a) that if both $\mathrm{v}$ and $\Delta \mathrm{x}$ are non-vanishing, the result is that $\Delta \mathrm{t} \neq \Delta \mathrm{t}^{\prime}$. The problem is that this inequality also holds if $\Delta \mathrm{t}=0$. As a consequence, one is forced by the LT to believe in remote non-simultaneity, i.e. that events which occur simultaneously for one observer $(\Delta t=0)$ need not do so for anyone else in relative motion to him $\left(\Delta t^{\prime} \neq 0\right)$. This state of affairs seems first to have been noticed by Poincaré in 1898 [8, 9]. He argued that such an unusual situation could not be ruled out on the basis of existing experiments which were available at that time. He also recognized that remote non-simultaneity was completely foreign to what physicists had believed for the preceding 200 years based on the position taken by Newton and his contemporaries toward the end of the $17^{\text {th }}$ century.

Einstein gave his own derivation of the LT in his landmark paper published in 1905 [10]. He also noted that remote non-simultaneity is an unavoidable consequence of the LT. In the same paper, however, he pointed out something else about expected elapsed time observations that is predicted by the LT, namely proportional time dilation.

The latter result can be obtained directly from eq. (6) by considering the following example [11]. In this case the object of the measurements is a stationary clock in one of the rest frames. From the point of view of an observer in the other rest frame, the distance $\Delta \mathrm{x}$ travelled by this clock satisfies the relationship: $\Delta \mathrm{x}=\mathrm{v}$ $\Delta \mathrm{t}(\Delta \mathrm{y}=\Delta \mathrm{z}=0)$. Since the clock is not moving for the other (primed) observer, it follows from eq. (6) that

$\left(\mathrm{v}^{2} \Delta \mathrm{t}^{2}-\mathrm{c}^{2} \Delta \mathrm{t}^{2}\right)=-\mathrm{c}^{2} \Delta \mathrm{t}^{2}$

Dividing through by $-\mathrm{c}^{2}$ and taking the square root on both sides therefore leads to the above result of proportional time dilation:
$\Delta \mathrm{t}^{\prime}=\gamma^{-1} \Delta \mathrm{t}$

The contradiction imposed on relativity theory by the LT because of its joint predictions of proportional time dilation and remote non-simultaneity can be easily demonstrated by means of a simple algebraic exercise which has been referred to in earlier work [12] as the Clock Puzzle. Consider the following pair of equations involving two variables $\Delta \mathrm{t}$ and $\Delta \mathrm{t}^{\prime}$. They are to be associated with the time differences separating lightning strikes in a forest or on a moving train measured by two observers who are in relative motion to one another:

$$
\begin{aligned}
& \Delta \mathrm{t}=0 \\
& \Delta \mathrm{t}^{\prime}=\mathrm{X} \Delta \mathrm{t} .
\end{aligned}
$$

In the latter equations the quantity $\mathrm{X}$ can take on any finite value. According to the rules of elementary algebra, there is a unique solution for $\Delta \mathrm{t}^{\prime}$ based on these equations, namely $\Delta \mathrm{t}^{\prime}=0$.

To assume otherwise requires that one violate the axiom that states that multiplication of $\Delta \mathrm{t}=0$ in eq. (9b) by any number must result in a product of zero for $\Delta \mathrm{t}^{\prime}$.

The quantity $\mathrm{X}$ can be associated with the proportional time-dilation factor $\gamma^{-1}$ in eq. (8). As a result one is forced to conclude that $\Delta \mathrm{t}=\Delta \mathrm{t}^{\prime}=0$, in clear contradiction to the claim of remote non-simultaneity based on the LT. In short, one can have remote nonsimultaneity or proportional time dilation but not both $[13,14]$.

The Clock Puzzle therefore proves unequivocally that the LT is not a physically viable space-time transformation. The GVT of eqs. (3a-3d) nonetheless remains a useful tool in the search for a suitable replacement for the LT in relativity theory. One way to see this is to form the velocity transformation from the GVT by dividing each of its three spatial variables $\Delta x^{\prime}, \Delta y^{\prime}$ and $\Delta z^{\prime}$ by $\Delta t^{\prime}$. The result is the relativistic velocity transformation (RVT) given below $\left(\mathrm{u}_{\mathrm{x}}{ }^{\prime}=\Delta \mathrm{x}^{\prime} / \Delta \mathrm{t}^{\prime}, \mathrm{u}_{\mathrm{x}}=\Delta \mathrm{x} / \Delta \mathrm{t}\right.$, etc. $)$ :

$$
\begin{aligned}
& \mathrm{u}_{\mathrm{x}}^{\prime}=\left(1-\mathrm{vu}_{\mathrm{x}} \mathrm{c}^{-2}\right)^{-1}\left(\mathrm{u}_{\mathrm{x}}-\mathrm{v}\right)=\eta\left(\mathrm{u}_{\mathrm{x}}-\mathrm{v}\right) \\
& \mathrm{u}_{\mathrm{y}}^{\prime}=\gamma^{-1}\left(1-\mathrm{vu}_{\mathrm{x}} \mathrm{c}^{-2}\right)^{-1} \mathrm{u}_{\mathrm{y}}=\eta \gamma^{-1} \mathrm{u}_{\mathrm{y}} \\
& \mathrm{u}_{\mathrm{z}}^{\prime}=\gamma^{-1}\left(1-\mathrm{vu}_{\mathrm{x}} \mathrm{c}^{-2}\right)^{-1} \mathrm{u}_{\mathrm{z}}=\eta \gamma^{-1} \mathrm{u}_{\mathrm{z}}
\end{aligned}
$$

The same set of equations is obtained when the analogous procedure is applied to both the LT and the original Voigt transformation of eqs. (1a-1d). The predictions of the RVT have an excellent record. They are in agreement with such key experimental observations as the aberration of starlight at the zenith [15] and the Fresnel light-drag effect [16]. The RVT is also in explicit agreement with the light-speed constancy requirement, as expected based on its close relationship to the GVT. It also is consistent with the $\mathrm{RP}$, as will be shown below.

The question is clearly how to use the GVT to arrive at the true relativistic space-time transformation. 
The self-contradiction inherent in the LT's treatment of the relationship between space and time must be removed. This must be done, however, in a way that remains consistent with the RP.

To approach this goal, it is important to recall that the clocks mentioned in the above argumentation regarding proportional time dilation and remote nonsimultaneity are assumed to be inertial, i.e. not under the influence of unbalanced external forces. As such, they come under the jurisdiction of Newton's First Law of Motion, the Law of Inertia. As a consequence, they are expected to move with constant speed and direction in each case. What about the properties of such clocks? Since no outside forces are present, it follows from the Law of Causality that they all should remain unchanged indefinitely. This includes the rates of these clocks.

It cannot be ruled out, however, that any two such clocks do not have the same rate. What one can say with certainty on this basis, however, is that the ratio of inertial clock rates must also be constant. As a consequence, one therefore must expect that the elapsed

$$
\begin{aligned}
\Delta \mathrm{t}^{\prime}=\mathrm{Q}^{-1} \Delta \mathrm{t} & \\
\Delta \mathrm{x}^{\prime} & =\eta \mathrm{Q}^{-1}(\Delta \mathrm{x}-\mathrm{v} \Delta \mathrm{t}) \\
\Delta \mathrm{y}^{\prime} & =\eta(\mathrm{Q} \gamma)^{-1} \Delta \mathrm{y} \\
\Delta \mathrm{z}^{\prime} & =\eta(\mathrm{Q} \gamma)^{-1} \Delta \mathrm{z}
\end{aligned}
$$

By construction, eq. (11) is part of the above transformation by virtue of its eq. (14a). It clearly runs completely counter to the theory of remote nonsimultaneity because it only allows a null value for one of the time differences when the other is equal to zero itself. It is perfectly in line with the solution of the Clock Puzzle. It is also "Newtonesque" in its view of the separation of space and time that is otherwise espoused by the LT. It does ascribe to proportional time dilation, but this version is quite distinct from that predicted by the LT. The latter is "symmetric" in

$$
\begin{aligned}
& \Delta \mathrm{t}=\mathrm{Q}^{\prime-1} \Delta \mathrm{t}^{\prime} \\
& \Delta \mathrm{x}=\eta^{\prime} \mathrm{Q}^{\prime-1}\left(\Delta \mathrm{x}^{\prime}+\mathrm{v} \Delta \mathrm{t}^{\prime}\right) \\
& \Delta \mathrm{y}=\eta^{\prime}\left(\mathrm{Q}^{\prime} \gamma\right)^{-1} \Delta \mathrm{y}^{\prime} \\
& \Delta \mathrm{z}=\eta^{\prime}\left(\mathrm{Q}^{\prime} \gamma\right)^{-1} \Delta \mathrm{z}^{\prime} .
\end{aligned}
$$

As usual, in order to satisfy the RP, the latter set of equations must be the inverse of the original in eqs. (14a-14d). Substitution of eq. (16a) into eq. (14a) shows that there is a simple condition to be met in this case in order to satisfy the RP:

$$
\begin{aligned}
& \Delta \mathrm{t}^{\prime}=\mathrm{Q}^{-1} \Delta \mathrm{t}=\mathrm{Q}^{-1} \mathrm{Q}^{-1} \Delta \mathrm{t}^{\prime}, \\
& \text { namely, } \\
& \mathrm{QQ}^{\prime}=1 .
\end{aligned}
$$

The constant Q' must be the reciprocal of $\mathrm{Q}$. This requirement is perfectly understandable from the way in which $Q$ is defined in the above argument based on the Law of Inertia. The latter serves as a conversion times or other time differences satisfy the simple proportionality relation given below:

$$
\Delta \mathrm{t}^{\prime}=\mathrm{Q}^{-1} \Delta \mathrm{t}
$$

where $Q$ is the ratio of the respective clock rates mentioned above.

It is clear how eq. (11) can be combined with the GVT of eqs. (3a-3d). One simply has to assume that the two values of $\Delta \mathrm{t}^{\prime}$ in eq. (3a) and eq. (11) are equal, hence:

$\Delta \mathrm{t}^{\prime}=\chi\left(\Delta \mathrm{t}-\mathrm{vc}^{-2} \Delta \mathrm{x}\right)=\chi \eta^{-1} \Delta \mathrm{t}=\mathrm{Q}^{-1} \Delta \mathrm{t}$,

from which a unique value for $\chi$ is determined to be $\chi=\eta Q^{-1}$.

This value for the parameter $\chi$ can then be substituted in the GVT to obtain a different space-time transformation [12-14, 17] than either the LT or the original Voigt transformation of eqs. (1a-1d):

nature. This can be seen by applying Galilean inversion to eq. (8). The result is:

$\Delta \mathrm{t}=\gamma^{-1} \Delta \mathrm{t}^{\prime}$.

It is therefore completely ambiguous [18] from these two equations which of two clocks in motion run slower. The situation is fundamentally different when Galilean inversion is applied to the transformation of eqs. (14a-d). In this case the result is:

factor between the respective timing results in the two rest frames, whereas $\mathrm{Q}^{\prime}$ is the corresponding conversion factor in the reverse direction.

Does one need a separate condition for the other three equations in order for them to satisfy the RP as well? To judge this, consider the case for the $y$ coordinate based on eqs. (14c) and (16c):

$$
\Delta y^{\prime}=\eta(Q \gamma)^{-1} \Delta y=\eta(Q \gamma)^{-1} \eta^{\prime}\left(Q^{\prime} \gamma\right)^{-1} \Delta y^{\prime} .
$$

After taking eq. (18) into account, one is left with $\eta \eta^{\prime}\left(Q^{\prime}\right)^{-1} \gamma^{-2}=\eta \eta^{\prime} \gamma^{-2}=1$ 
as the remaining condition. It is automatically satisfied by using the previous definitions of $\gamma, \eta$ and $\eta^{\prime}$. The inverse of the $\eta \eta^{\prime}$ product is used to demonstrate the latter statement. The result is $\gamma^{-2}$, consistent with eq. (20). Note that eq. (10a) of the RVT is used in the process:

$$
\begin{gathered}
\left(\eta \eta^{\prime}\right)^{-1}=\left(1-\mathrm{u}_{\mathrm{x}} \mathrm{vc} \mathrm{c}^{-2}\right)\left(1+\mathrm{u}_{\mathrm{x}}^{\prime} \mathrm{vc} \mathrm{c}^{-2}\right)=\mathrm{c}^{-4}\left(\mathrm{c}^{2}-\mathrm{u}_{\mathrm{x}} \mathrm{v}\right)\left(\mathrm{c}^{2}+\mathrm{u}_{\mathrm{x}}^{\prime} \mathrm{v}\right) \\
=\mathrm{c}^{-4}\left(\mathrm{c}^{2}-\mathrm{u}_{\mathrm{x}} \mathrm{v}\right)\left[\mathrm{c}^{2}+\eta\left(\mathrm{u}_{\mathrm{x}}-\mathrm{v}\right) \mathrm{v}\right]=\mathrm{c}^{-2}\left(\mathrm{c}^{2}-\mathrm{u}_{\mathrm{x}} \mathrm{v}\right)\left(\mathrm{c}^{2}-\mathrm{u}_{\mathrm{x}} \mathrm{v}+\mathrm{u}_{\mathrm{x}} \mathrm{v}-\mathrm{v}^{2}\right)\left(\mathrm{c}^{2}-\mathrm{u}_{\mathrm{x}} \mathrm{v}\right)^{-1} \\
=1-\mathrm{v}^{2} \mathrm{c}^{-2}=\gamma^{-2} .
\end{gathered}
$$

The analogous procedure clearly also works for the $\Delta z^{\prime}$ component. The situation for $\Delta x^{\prime}$ is somewhat more complicated, but the desired result is also obtained in this case. The explicit derivation given below is for $u_{x}$, demonstrating that the RVT also satisfies the RP.

$$
\begin{gathered}
\mathrm{u}_{\mathrm{x}}=\eta^{-1} \mathrm{u}_{\mathrm{x}}^{\prime}+\mathrm{v}=\eta^{\prime} \gamma^{-2} \mathrm{u}_{\mathrm{x}}{ }^{\prime}+\left(\eta^{\prime} \eta^{\prime-1}\right) \mathrm{v} \\
=\eta^{\prime}\left[\mathrm{u}_{\mathrm{x}}^{\prime}-\mathrm{v}^{2} \mathrm{c}^{-2} \mathrm{u}_{\mathrm{x}}^{\prime}+\mathrm{v}\left(1+\mathrm{u}_{\mathrm{x}}^{\prime} \mathrm{vc}^{-2}\right)\right] \\
=\eta^{\prime}\left(\mathrm{u}_{\mathrm{x}}{ }^{\prime}+\mathrm{v}\right)=\mathrm{u}_{\mathrm{x}} .
\end{gathered}
$$

The inverse transformation for the RVT has been used in this case. The latter is also obtained by applying Galilean inversion to the original in eqs. (10a$10 \mathrm{c})$. It should be noted that the RP is automatically satisfied by the RVT even though its derivation only assumes the light-speed constancy requirement.

An alternative route to eqs. (14a-14d) consists of first forming the RVT from the original Voigt transformation in eqs. (1a-1d) and then combining it with eq. (11) by simple multiplication of each of the three RVT equations. In either case the result is a relativistic space-time equation that satisfies both of Einstein's postulates [10], the RP and light-speed constancy, but eliminates the problematic space-time mixing inherent in the LT which is responsible for the contradiction unveiled by the Clock Puzzle. It has previously been referred to as the Alternative Lorentz Transformation [13] or as the Global Positioning System -Lorentz Transformation (GPS-LT) [12, 14, 19, 20]. The present discussion indicates that it would be perhaps more aptly named the Newton-Voigt Transformation (NVT) because of its derivation from eqs. (1a-d) in combination with eq. (11).

\section{Iv. Experimental tests and the universal time- dilation law}

One of the first things that comes to mind when it is realized that the LT is invalid is how it could be that physicists espoused it for such a long time. The simple answer is that it was found that the LT is consistent with a wide variety of experiments for which an alternative interpretation was seemingly non-existent and this fact made it extremely unlikely that it could be super-ceded by a different transformation. Perhaps the main explanation for this state of affairs is that many of the key experimental successes attributed to the LT are actually due to the RVT of eqs. (10a-10c), which in turn can be derived from any of a number of other sets of equations such as the original Voigt transformation of eqs. (1a-1d), the GVT of eqs. (3a-3d) and the NVT of eqs. (14a-14d) in the same way as from the LT itself. For example, the key result of aberration of starlight at the zenith [15], which is so critical for astronomical observations, has been derived on the basis of the LT alone [21], whereas the same result can be obtained from the RVT in a notably simpler manner [22]. It was shown for the first time by von Laue [16] two years after Einstein's work [10] had appeared that the Fresnel light-drag experiment can be completely elucidated on the basis of one of the RVT equations. All the experimental investigations that invariably confirm Einstein's velocity addition theorem, which shows among other things that $\mathrm{c}$ is the limiting value of the speed of light in free space, are correctly interpreted by using the RVT exclusively [23]. One also does not need the LT to derive the Thomas spin precession of the electron [24] since the only observable quantity also involves a ratio in which the time coordinate is present in the denominator [25].

In order to find experiments whose results are in conflict with LT predictions, it is therefore necessary that either elapsed times or distances be measured separately, not just ratios of same. The first such experiments were carried out in the late 1930s. Ives and Stilwell [26] investigated the transverse Doppler effect which had been predicted by Einstein 33 years earlier [10]. Their conclusion was that the frequency of light whose source had been accelerated to speed $\mathrm{v}$ in their laboratory was decreased by a factor of $\gamma(\mathrm{v})$ relative to its standard value. This result was seen to be consistent with Einstein's prediction based on eq.(8) of time dilation in the rest frame of the light source.

The experiment carried out by Ives and Stilwell did not measure the frequency of the radiation, however, but rather its wavelength. It was found that 
the latter's value measured on a photographic plate at rest in the laboratory was $\gamma(\mathrm{v})$ times larger than the standard value. This result stands in conflict with another prediction of his theory, however, namely the FitzGerald-Lorentz length contraction effect (FLC). Accordingly, it would be expected that the wavelength be the same in the laboratory as in the accelerated rest frame because it was measured in a direction transverse to that of the light source. No mention was made of this aspect of the experiment in the original paper [26]. It was simply concluded that the increase in wavelength indicated that a decrease in frequency by the same factor $\gamma(\mathrm{v})$ had occurred because of the light-speed constancy assumption of the theory.

A similar result was obtained at about the same time [27] in a study of accelerated muons. What was actually measured was again a distance quantity, in this case the length of the average decay path of the accelerated particles moving with speed $\mathrm{v}$ relative to the laboratory. The fact that this distance was equal to $\gamma(\mathrm{v})$ $\mathrm{v} \tau$ instead of $\mathrm{v} \tau$ was then widely interpreted to be proof of an increase in the lifetime $\tau$ of the muons to $\gamma(\mathrm{v}) \tau$ caused by their acceleration, again as expected from eq. (8). The FLC, on the other hand, predicts that the average decay path of the muons should be smaller or equal to $v \tau$ depending on their orientation relative to the laboratory.

This discrepancy between theory and experiment has simply been falsely interpreted in textbooks $[28,29]$. It is easy to see what the correct relationship between elapsed times and distances of travel should be based on the following example. A measurement of the distance $\mathrm{L}$ light moves in time $\mathrm{T}$ is found to be cT. The laboratory is then accelerated to speed $\mathrm{v}$ and the experiment is repeated. The observer $\mathrm{M}$ in the accelerated laboratory, in accordance with the $\mathrm{RP}$, still finds that the elapsed time for the light to travel the distance $\mathrm{L}$ is equal to cT. However, his counterpart $\mathrm{N}$ left behind in the original rest frame now finds that the elapsed time in the accelerated laboratory has increased because of time dilation in the latter rest frame to a value of $\gamma \mathrm{T}$. Since the speed of light in M's rest frame is also equal to $\mathrm{c}$ for observer $\mathrm{N}$, it follows that the distance traveled has increased for him to a value of $\mathrm{c}(\gamma \mathrm{T})=\gamma \mathrm{cT}=\gamma \mathrm{L}$. Since $\mathrm{N}$ has been at rest for the entire experiment, the only reasonable conclusion is that the distance in M's rest frame has also increased by a factor of $\gamma(\mathrm{v})$. The conclusion, which is clearly based on the light-speed constancy assumption, is that lengths expand in the same proportion as clocks slow down in a given rest frame. It is nonetheless in clear opposition to the prediction of the FLC, which in turn is based directly on the LT.

The experiments discussed above employ measurements of the properties of accelerated objects from the vantage point of an observer who is at rest in the corresponding laboratory. As a result they are incapable of testing one of the most famous LT predictions, symmetric time dilation. As discussed above, two distinct formulas can be derived: eqs. (8) and (15). They are related by Galilean inversion on the one hand, but on the other, they are clearly not the inverse of one another, which means that they are inconsistent with the RP. This result in itself is curious because of the fact that the LT itself does satisfy the RP, and yet equations derived from it do not.

Nonetheless, it can be shown from the way that eqs. (8) and (15) are derived that it is always the "moving" clock that runs slower from the perspective of the observer in the other rest frame. This interpretation is therefore consistent with what is observed from the laboratory perspective in each of the transverse Doppler [26] and muon decay [27] studies, since they both indicate that it is the accelerated "clock" that runs slower than the corresponding laboratory clock.

The first experiment which was in a position to test whether time dilation is symmetric or not was carried out by Hay et al. in 1960 [30]. An x-ray source and detector were mounted on a high-speed rotor and the change in frequency with the speed of the rotor was measured. The experiment was subsequently repeated by Kuendig [31] and Champeney [32], with essentially the same results.

What was found is that the frequency observed at the detector increases relative to the standard value emitted from the source in direct proportion to $\gamma(\mathrm{R} \omega)$, where $\mathrm{R}$ is the radius of the rotor and $\omega$ is the rotational frequency of the rotor. Hay et al. claimed that this result was fully consistent with the LT prediction of symmetric time dilation, but it clearly is not because the results show that from the perspective of the detector mounted on the rim of the rotor, which is therefore moving with high speed relative to the $\mathrm{x}$-ray source mounted on the axis, the frequency of radiation coming from the latter is measured to be greater, not less, than the standard value. This means that from the vantage point of the accelerated "observer" at the rim of the rotor, it is his clock that runs slower. That is asymmetric time dilation, not the symmetric result expected on the basis of eqs. (8) and (15). Kuendig [31] made this quite clear in his description of the experiment: "It is always the accelerated clock that runs slower." In other words, it doesn't matter who is doing the observing, everyone agrees that the latter clock is slower than one moving at a lower rotational speed. An attempt [30-32] was made to explain this result by invoking Einstein's Equivalence Principle between kinetic and gravitational acceleration [33]. Sherwin argued instead that the key point in the rotor experiments was that it is not ambiguous which clock is running slower, and that this result clearly runs counter to what is expected on the basis of the LT [34]. He suggested that the symmetric form of time dilation would be observed were it not for the fact that the $\mathrm{x}$-ray detector is undergoing acceleration in the experiment, 
that things would be different if both the source and detector were inertial systems.

Ten years later a definitive test of the effects of motion on the rates of clocks was carried out by Hafele and Keating [35-36]. They carried newly invented "atomic clocks" on board circumnavigating airplanes. Their results indicated a clear relationship between the speed of a given clock relative to the earth's center of gravity (ECM). The most obvious consequence was that the eastward-flying clocks ran slower than their counterparts left behind at the airport of origin, which in turn ran slower than the other clocks flying in a westerly direction around the globe. Another key result was their finding that the effects of gravity on the respective clock rates could be taken quantitatively into account by using Einstein's gravitational red shift formula [33]. When the latter adjustment was made, it was found that the rates of the clocks were inversely proportional to their speed relative to the ECM. The effect on measured, red-shift adjusted, elapsed times $\Delta t$ and $\Delta \mathrm{t}^{\prime}$ for two clocks moving, respectively, with speeds $\mathrm{v}$ and $\mathrm{v}$ ' relative to the ECM is given below:

$\Delta \mathrm{t}^{\prime} \gamma\left(\mathrm{v}^{\prime}\right)=\Delta \mathrm{t} \gamma(\mathrm{v})$

The above formula accounts quantitatively (within error bars of $10 \%$ [36]) for the aforementioned east-west asymmetry in the HK elapsed time data: an airplane traveling with speed $\mathrm{w}$ in an easterly direction has a higher speed relative to the ECM, and therefore a lower clock rate according to eq. (23), than either a stationary position at the airport or an airplane traveling in the opposite direction with the same ground speed w.

The above results clearly stand in direct conflict with the LT prediction of symmetric time dilation. They also run contrary to the Equivalence Principle argument mentioned above in connection with the rotor experiments [31-34], since gravitational effects on the clock rates are explicitly removed from consideration in obtaining the empirical formula given in eq. (23). There is also independent evidence from other experiments [37] to indicate that the degree of acceleration has no effect on clock rates. Moreover, unlike the case for the rotor studies, the clocks on the airplanes were essentially moving with constant speed for almost the entire duration of the flights [36], rendering them to be "inertial systems' for all practical purposes.

Another key failure of the LT is its prediction based on eqs. (8) and (15) that the amount of time dilation on the clocks is determined exclusively by the relative speed $v$ of the two observers. Instead, it is the speed of each clock relative to the ECM that is ratedetermining. This is an especially important result because it shows that measurement is not a subjective process, depending on one's own perspective as Einstein would have us believe [10], but rather perfectly objective. The same conclusion can be reached on the basis of the rotor study [31], as already mentioned.

What the HK results show is that one must first identify a specific rest frame from which to evaluate the relevant speeds to be inserted in the formula for time dilation. The latter has been referred to in previous work as the objective rest system (ORS) [38]. Once this is recognized, it is possible to use eq. (23) to obtain the corresponding timing comparisons in the rotor study, in which case the ORS is the axis of the rotor, or the rest frame of the laboratory in which the $\mathrm{x}$ ray frequency measurements were made. The same equation is applicable to the "clock paradox" discussed by Einstein in which he compared the rate of a clock attached to an electron moving in a circular path to that left behind at the origin. In that case, the ORS is the position where a force was applied to the electron in order to induce its acceleration. The same designation should apply to any object undergoing acceleration as a result of an applied external force, in accord with Newton's Second Law of Motion.

Given the above results, it is reasonable to look upon eq. (23) as the Universal Time-dilation Law (UTDL) [39]. To apply this formula, it is first necessary to identify the appropriate ORS for determining the speeds $\mathrm{v}$ and $\mathrm{v}^{\prime}$. In practice, one might need distinct ORSs, for example, when comparing the rate of a clock orbiting the moon to one in the earth's gravitational field.

There is a clear relationship between the UTDL and the NVT. One simply must connect eq. (23) with eq. $(14 a, 11)$ of the latter, in which case $Q$ is fully determined [40]:

$$
\mathrm{Q}=\Delta \mathrm{t} / \Delta \mathrm{t}^{\prime}=\gamma\left(\mathrm{v}^{\prime}\right) / \gamma(\mathrm{v})
$$

Applying Galilean inversion to eq. (24), one obtains the correct result for $\mathrm{Q}^{\prime}$ as:

$$
\mathrm{Q}^{\prime}=\Delta \mathrm{t}^{\prime} / \Delta \mathrm{t}=\gamma(\mathrm{v}) / \gamma\left(\mathrm{v}^{\prime}\right),
$$

Which is clearly consistent with eq. (18) required for satisfaction of the RP by the NVT.

\section{UNIFORM SCALING OF PHYSICAL UNITS}

The most insightful way to look upon the parameter $Q$ in the NVT set of equations is as a conversion factor between the elapsed times for the same event measured by two observers in relative motion to one another. It means, as shown explicitly in eq. (24), that the value $\Delta \mathrm{t}^{\prime}$ obtained by one observer need only be multiplied by $\mathrm{Q}$ in order to exactly deduce the corresponding value $\Delta \mathrm{t}$ by his counterpart in the other rest frame. As such, Q plays the same role as conversion factors for $\mathrm{cm}$ and $\mathrm{ft}$ or $\mathrm{N}$ with $\mathrm{lb}$ in everyday life. The same relationship also holds for the conversion factor Q' in eq. (25) between elapsed times in the reverse direction, whereby the value in the 
forward direction is simply seen to be the reciprocal of that in the other, as required to satisfy the RP [see eq. (18)]. More generally, such a reciprocal relationship is an obvious requirement from the very definition of conversion factors.

The conclusion that the same conversion factor can be used for the elapsed times of any event is directly relevant to the Relativity Principle (RP). If there were two events for which a different conversion factor for their elapsed times was needed in each case, this would mean that the ratio of these two quantities would be different in one rest frame than in the other. That in turn would allow an observer in one rest frame to be able to distinguish his state of free translation from that of another by simply carrying out an in situ measurement of this ratio, something that is expressly ruled out by the RP. Einstein used the same argument to analyze frequency measurements in different locations in a gravitational field in order to derive the formula for the gravitational red-shift [33, 41].

The parameter Q in the NVT of eqs. (14a-14d) also serves as a conversion factor for distances. This is because the speed of light in free space is the same in all rest frames. As discussed in Sect. IV, the distance traveled by light in a given rest frame increases by exactly the same fraction as the corresponding elapsed time as the state of motion is varied. As a consequence, there is a completely analogous relation for measured distances as for elapsed times in the UTDL of eq. (23).

A simple way of expressing these relations is to say that both distance and time scale as Q. At the same time, it follows that relative velocities that is the velocity by which two objects separate as a result of their motion, is the same for all observers. The latter statement follows directly from the light-speed constancy requirement [42]. One can therefore also express this result in terms of the conversion factor $\mathrm{Q}$ for elapsed times and distances traveled by saying that velocity scales as $\mathrm{Q}^{0}$. Moreover, the experiments carried out by Buecherer [43] indicate that the inertial mass of accelerated electrons also increases in direct proportion to $\gamma(\mathrm{v})$, whereupon one can say with equal justification that there is a corresponding inverse relation as the UTDL for inertial masses, and that these quantities also scale with the same value of $Q$ as elapsed times and distances traveled.

Once the conversion factors for distance, inertial mass and time are in hand, it is a simple matter to form the analogous quantities for all other physical properties. All one needs to know is the composition of each in terms of the latter three fundamental units. The result is therefore always $\mathrm{Q}$ raised to an integral exponent. For example, energy $\mathrm{E}$ also scales as $\mathrm{Q}$ because of its composition of inertial mass and the square of a velocity. Angular momentum 1 scales as $Q^{2}$ because it is the product of mass, speed and distance.
The same therefore also holds true for Planck's constant $\mathrm{h}$, since it has the same dimensions as 1 . This explains why the Planck Radiation Law $(E=h v)$ holds in all rest frames despite the fact that $\mathrm{E}$ scales as $\mathrm{Q}$ but the frequency $v$, since it is the reciprocal of the period of the radiation, scales as $\mathrm{Q}^{-1}$. In other words, unlike $\mathrm{c}, \mathrm{h}$ varies with its state of motion.

The general procedure for determining the way in which the properties of objects change with their state of motion is referred to as uniform scaling. More details regarding this subject may be found elsewhere $[44,45]$. It is even possible to fit electromagnetic quantities into this scheme [46, 47]. Finally, it should be noted that there is an equivalent procedure involving a different quantity $S$ which can be applied to the scaling of properties with regard to their position in gravitational field. The latter methods are perfectly consistent with the calculations of elapsed times carried out by Hafele and Keating in their experiments with circumnavigating atomic clocks $[35,36]$.

\section{CONCLUSION}

For over a century it has been the widespread belief of physicists that space-time mixing is essential in order to modify the classical Galilean transformation to be consistent with experiments that demonstrate that the speed of light in free space is independent of both the state of motion of the observer and the light source. The alternative transformation that Voigt published in 1887 is the first example in which this idea was put into practice. The latter set of equations failed to satisfy the Relativity Principle (RP), however, and was therefore rejected.

The irony is that the Voigt transformation can be used directly to obtain the long-accepted Relativistic Velocity Transformation (RVT) of eqs. (10a-10d) by simply forming the necessary space/time ratios from it. That being the case, all that was needed to correct the situation was to realize that clocks moving without the influence of unbalanced external forces must have constant rates. The only way to satisfy this basic requirement, which is closely related to Newton's First Law of Motion, is to insist that space and time are not mixed. As a consequence, elapsed times obtained by two observers in relative motion for the same event must be directly proportional to one another, as indicated in eq. (11).

Combining eq. (11) with the RVT leads to the Newton-Voigt space-time transformation (NVT) of eqs. $(14 \mathrm{a}-14 \mathrm{~d})$. It is fundamentally different than the Lorentz Transformation (LT) of eqs. (5a-5d). The NVT not only satisfies both the requirements of light-speed constancy and proportional time dilation, but it also conforms to the RP. Unlike the LT, however, the NVT is not consistent with remote non-simultaneity. It therefore avoids the contradiction with proportional time dilation exposed by the Clock Puzzle of eqs. (9a-9b). 
The experimental results obtained in studies of time dilation can be summarized in terms of the inverse proportionality relation of eq. (23), which is referred to as the Universal Time-dilation Law (UTDL). It can be used directly via eq. (24) to determine the proportionality constant $\mathrm{Q}$ in the NVT. To do so, however, it is necessary to identify a specific rest frame (objective rest system, ORS) relative to which the speeds in the UTDL are defined. For example, the ORS is the axis of the rotor in the 1960 Hay et al. study of xray frequencies, whereas it is the earth's center of mass (ECM) in the Hafele-Keating experiment with circumnavigating atomic clocks. Notably, the relative speed of the two observers is not needed in applying the UTDL, contrary to the case in the symmetric theory of time dilation predicted by the LT, according to which it is purely a matter of perspective which of the two clocks runs slower. By contrast, the NVT and UTDL are consistent with a strictly objective view of measurement, one which asserts that the results of experiments are not simply a matter of perspective.

The parameter Q in the NVT can be looked upon as a conversion factor between elapsed times measured for the same event by two observers in different rest frames. The same conversion factor holds for distances and inertial masses. The corresponding quantities for all other physical quantities, including those which are electromagnetic in nature, can be determined to be integral powers of $\mathrm{Q}$ based on the composition of the latter in terms of the above three fundamental quantities.

The procedure for relating experimental measurements in different rest frames is referred to as uniform scaling. This uniformity in nature is a necessary consequence of the RP. An analogous scaling procedure exists for the properties of objects measured by observers located in different positions in a gravitational field.

\section{REFERENCES}

1. Michelson, A. A., \& Morley, E. W. (1887). On the Relative Motion of the Earth and of the Luminiferous Ether. Sidereal Messenger, vol. 6, pp. 306-310, 6, 306-310.

2. Pais, A. (2005). Subtle is the Lord. The Science and Life of Albert Einstein, 1982.

3. Voigt, W. (1887). Ueber das Doppler'sche Princip. Nachr Ges Wiss, 41.

4. Lorentz, H. A. (1902). De draaiing van het polarisatievlak in lichamen die zich bewegen. Versl. K. Ak. Amsterdam, 10, 793.

5. Pais, A. (2005). Subtle is the Lord. The Science and Life of Albert Einstein, 1982.

6. Lorentz, H. A. (1937). Electromagnetic phenomena in a system moving with any velocity smaller than that of light. In Collected Papers (pp. 172-197). Springer, Dordrecht.
7. Larmor, J. (1900). Aether and Matter, Cambridge University Press, Cambridge,

8. Poincaré, H. (1898). Rev. Métaphys. Morale 6,1.

9. Pais, A. (2005). Subtle is the Lord. The Science and Life of Albert Einstein, 1982.

10. Einstein, A. (1905). Zur elektrodynamik bewegter körper. Annalen der physik, 4.

11. Jackson, J. D. (1962). Classical Electrodynamics, John Wiley and Sons, New York, 358-359.

12. Buenker, R. J. (2018). The clock puzzle and the incompatibility of proportional time dilation and remote non-simultaneity.

13. Buenker, R. J. (2008). Apeiron 15, 254.

14. Buenker, R. J. (2018). The Spacetime Myth: Failure of the Lorentz Transformation.

15. Pais, A. (2005). Subtle is the Lord. The Science and Life of Albert Einstein, 1982.

16. M. von Laue, Ann.(1907). Physik 23, 989.

17. Buenker, R. J. (2018). Voigt's Conjecture of Spacetime Mixing: Contradiction between Nonsimultaneity and the Proportionality of Time Dilation.

18. Sherwin, W. (1960). Phys. Rev. 120, 17.

19. Buenker, R. J. (2014). Relativity Contradictions Unveiled: Kinematics, Gravity and Light Refraction jpf.

20. Buenker, R. J. (2015). GPS-compatible Lorentz transformation that satisfies the Relativity Principle. J. Astrophys. Aerospace Technol, 3(115), 1-6.

21. Kilmister, C. W. (1970). Special Theory of Relativity, Pergamon Press, Oxford,

22. Buenker, R. J. (2014). Relativity Contradictions Unveiled: Kinematics, Gravity and Light Refraction jpf.

23. Sard, R. D. (1970). Relativistic Mechanics, W. A. Benjamin, New York, 104-111.

24. Sard, R. D. (1970). Relativistic Mechanics, W. A. Benjamin, New York, 285-290.

25. Buenker, R. J. (2014). Relativity Contradictions Unveiled: Kinematics, Gravity and Light Refraction jpf.

26. Ives, H. E., \& Stilwell, G. R. (1938). An experimental study of the rate of a moving atomic clock. JOSA, 28(7), 215-226.

27. Rossi, B., Greisen, K., Stearns, J. C., Froman, D. K., \& Koontz, P. G. (1942). Further measurements of the mesotron lifetime. Physical Review, 61(1112), 675 .

28. Weidner, R. T., \& Sells, R. L. (1960). Elementary modern physics.

29. Serway, R. A., \& Beichner, R. J. (1999). Physics for Scientists and Engineers, $5^{\text {th }}$ Edition Harcourt, Orlando, 1262.

30. Hay, H. J., Schiffer, J. P., Cranshaw, T. E., \& Egelstaff, P. A. (1960). Measurement of the red shift in an accelerated system using the Mössbauer effect in Fe 57. Physical Review Letters, 4(4), 165.

31. Kündig, W. (1963). Phys. Rev. 129, 2371. 
32. Champeney, D. C., Isaak, G. R., \& Khan, A. M. (1963). Nature 198, 1186;1963.

33. Einstein, A. (1907). Jahrb. Rad.Elektr. 4, 411.

34. Sherwin, W. (1960). Phys. Rev. 120, 17.

35. Hafele, J. C., \& Keating, R. E. (1972). Science 177, 166.

36. Rindler, W. (1977). Essential Relativity, Springer Verlag, New York. 56-57.

37. Buenker, R. J. (2010). Apeiron 17, 99-125.

38. Buenker, R. J. (2014). Relativity Contradictions Unveiled: Kinematics, Gravity and Light Refraction jpf.

39. Buenker, R. J. (2014). Relativity Contradictions Unveiled: Kinematics, Gravity and Light Refraction, Apeiron, Montreal, 56.

40. Pais, A. (1982). 'Subtle is the Lord...' The Science and Life of Albert Einstein, Oxford University Press, Oxford, 198-199.
41. Buenker, R. J. (2016). Apeiron 20,73-83.

42. Bucherer, A. H. (1908). Phys. Zeit. 9, 755.

43. Buenker, R. J. (2018). Apeiron, 20(4), 1-31.

44. Buenker, R. J. (2014). Relativity Contradictions Unveiled: Kinematics, Gravity and Light Refraction jpf.

45. Buenker, R. J., Found, J. (2015). Applic. Phys. 2, 11-16.

46. Buenker, R. J. (2014). Relativity Contradictions Unveiled: Kinematics, Gravity and Light Refraction jpf.

47. Buenker, R. J. (2008). Apeiron 15, 382-413.

48. Buenker, R. J. (2014). Relativity Contradictions Unveiled: Kinematics, Gravity and Light Refraction, Apeiron, Montreal, 113-120.

Cite This Article: Robert J. Buenker (2021). The Hafele-Keating Circumnavigating Atomic Clock Experiment and the Newton-Voigt Relativistic Space-time Transformation. East African Scholars J Eng Comput Sci, 4(3), 27-36. 